\title{
Central Locker System for shopping mall using NFC Based Smartphone
}

\author{
Siddarth Poddar \\ System LSI Group, Samsung R\&D Institute (SRI-B), Bangalore, India \\ s.poddar@samsung.com
}

\begin{abstract}
Depositing purchased item at each store's locker system and carrying a token in hand for the assigned locker while entering a store in a shopping mall as well as collecting the item back while exiting the store is a time consuming exercise and even long delayed if the token is lost. The time would even multiply visiting multiple numbers of stores in a mall. Sometimes waiting in a long queue for depositing item at the store is an irritating hassle and everyone wants to skip this hassle. This paper introduces a novel solution using NFC enabled Smartphone and NFC reader located at each checkout counter of the store which provides an easy and convenient way to immediately keep your purchased things safe at the centrally located locker system and move around openly. Locker number would be generated at the store checkout counter after purchasing the item and just a tap of smartphone on NFC reader save the locker number information on NFC application installed on smartphone, which is used to retrieve the item back while exiting the mall. With the proposed solution, usage of extra space for each store's locker system and the corresponding staff can be eliminated, leading to cost reduction, promote efficiency and enhance customer service experience.
\end{abstract}

Keywords-Near Field Communication, Host based card emulation, Shopping mall, Central locker system.

\section{Introduction}

Shopping is an activity which allows one or more retailers to present their goods and products to the customers to help them in selecting a suitable item according to their desire [1]. Shopping mall is providing a bigger platform, to have all the retailers, merchandisers at one place selling their products, and to the customers providing wide variety of products all at one place with different brands. It can be a very pleasant or awful shopping experience for customers depending on number of factors like their convenience, easy access to stores/outlets etc. These factors would be even more significant when it comes to those who are over particular or have shopping addiction. Customers have to carry purchased items in hand while moving from one store to another or even roaming inside the mall. Customer need to deposit the item at the counter of each store while moving in. Store guy provides a token for that to collect it back while exiting the store. This consumes a lot of time in order to deposit the item and to get it back. Some shopping malls have a system of central locker system, in which customer has been provided with a token at the checkout counter of the store only while purchasing the item which can be used to retrieve the item back while exiting the mall. It allows them to move openly and even saves a 
lot of time. Both such processes have at least one limitation; customer should not miss the token. To overcome these limitations would be greatly valuable for shopping mall business sector to provide better service experience to customer, helps in cost reduction, and better space utilization. This paper presents a novel solution using NFC technology [2]. NFC has already shown its capabilities in many areas like communication, social networking, entertainment and even in the field of shopping business like mobile payment, indoor navigation system, customer assistance etc. [3, 4] In addition to all these, one more application of NFC in shopping mall is the locker management system which helps the customer to move around openly without carrying any item in hand and not even the token inside the mall. This comprises of centrally located locker system at the entrance or at the exit door of the shopping mall, NFC enabled smartphone with customer, NFC reader [5] at the checkout counter of each store of the mall as well as at the Parcel Dispatch Station. The proposed solution starts with a tap of customer's NFC enabled smartphone on the NFC reader placed at the store checkout counter which stores the assigned locker number of his purchased items to the NFC application in the phone. The saved locker number is used to retrieve the item back from central locker system while exiting the mall.

\section{Near Field Communication (NFC)}

NFC technology is a bidirectional, contactless communication between two devices. It operates at RF frequency of $13.56 \mathrm{MHz}$ in a short range of $10 \mathrm{~cm}$ or even less with a data rate ranging from $106 \mathrm{kbit} / \mathrm{s}$ $424 \mathrm{kbit} / \mathrm{s}$ [2]. When the two NFC devices come in close proximity triggers the communication, and NFC is just a platform for communication between two devices. As the technology operates in a very limited range, it is ideal for secure transactions [6]; even it serves as a safeguard against hackers. NFC can operate in three communication modes:

a) Tag Read/Write mode;

b) Peer to Peer mode;

c) Card Emulation mode.

Card emulation mode enables NFC based smartphones to act as a contactless card or tag to interact with NFC reader. The card or tag application lives on some other piece of hardware like Embedded Secure Element (ESE), UICC etc. that is wired to the NFC controller [7]. A NFC based smartphone does not generate its own RF field; instead NFC reader generates this field. Supported RF interfaces are ISO/IEC 14443 Type A, ISO/IEC 14443 Type B and Felica. NFC reader can write any data to a tag/NFC device or read from it. Data can be any simple text message, or URL etc. NFC forum has suggested a common specification for all these type of data called NDEF format (NFC Data Exchange Format). NDEF specifications defines a message encapsulation format to exchange information, e.g. between an NFC Forum device and another NFC forum device or an NFC Forum tag [8]. Android 4.4 introduces an additional method of card emulation that does not involve a secure element, called hostbased card emulation (HCE). This allows any Android application to emulate a card and talk directly to the NFC reader. In this case, the data is routed to the host CPU on which Android applications are running directly, instead of routing the NFC protocol frames to a secure element.[9] The proposed solution operates in NFC host based card emulation mode using NFC based smartphone as a NFC tag to store the information and the NFC reader. NFC readers are to be placed at the store checkout counter as well as the parcel dispatch station. NFC reader will generate RF field and start sensing NFC capable devices in its vicinity. When mobile device is tapped on NFC reader, it senses the device and starts with the initialization and anti-collision process, and automatically activates the specific installed application on 
the device and store the data into it which would be visible to customer on screen to verify. At the time of exiting mall, customer is advised to tap the device again on NFC reader placed at the parcel dispatch station, which again automatically activates the specific installed application on the device. At this time, NFC reader will read the data stored in the application instead of writing. The valid data stored would be visible to customer on screen for verification. Customer needs to verify the items and Exit session on device. Even, Customer can check the locker numbers and other details anytime while opening the application installed on device.

\section{System Design}

A system design has been proposed in this paper for the customers having NFC enabled smartphone to store the information of the locker which will have purchased product of the customer. This Application is intended for deployment in Shopping Mall to be used by customers during shopping. It involves both the customer as well as the seller. This system design has only one assumption that the wireless connection (Wi-Fi) inside the shopping mall should be reliable and fast. It comprises of three components:

a) Customer's NFC enabled smartphone

b) Locker Generate system at each checkout counters of stores.

c) Parcel dispatch station at the central locker system of mall where the purchased items are to be kept.

\section{a) NFC Enabled Smartphone:}

The most important component of this solution is NFC enabled smartphone with customer. A couple of years ago there were no more than a handful of phones supporting NFC. Now, 9 out of 10 of the top ten manufacturers sell NFC phones - the exception being Apple [10]. Mobile device should have the specific application intended for this solution installed. If application is not installed already, then mobile device should be configured to redirect to the application web for downloading using Wi-Fi. The solution prototype is developed on the Android smartphone in eclipse using Java along with the libraries provided by the Android SDK [11]. Customer need to register first to get the username or user id which helps to maintain his data in the shopping mall database. Even it helps customers to check the details of assigned lockers anytime as well as the previous history also. The information stored in the application comprise of Store name, Assigned Locker, number of Packets, cash memo id and status. The information is stored in the two different structure in the mobile application based on the "status" information. If the status of entry is "In Store" which means the packets are there in the central locker system of the shopping mall will be stored in application under the button named as "My Locker". It can be used for convenient tracking of purchases. It is completely implementation specific and can be modified as per the need. 


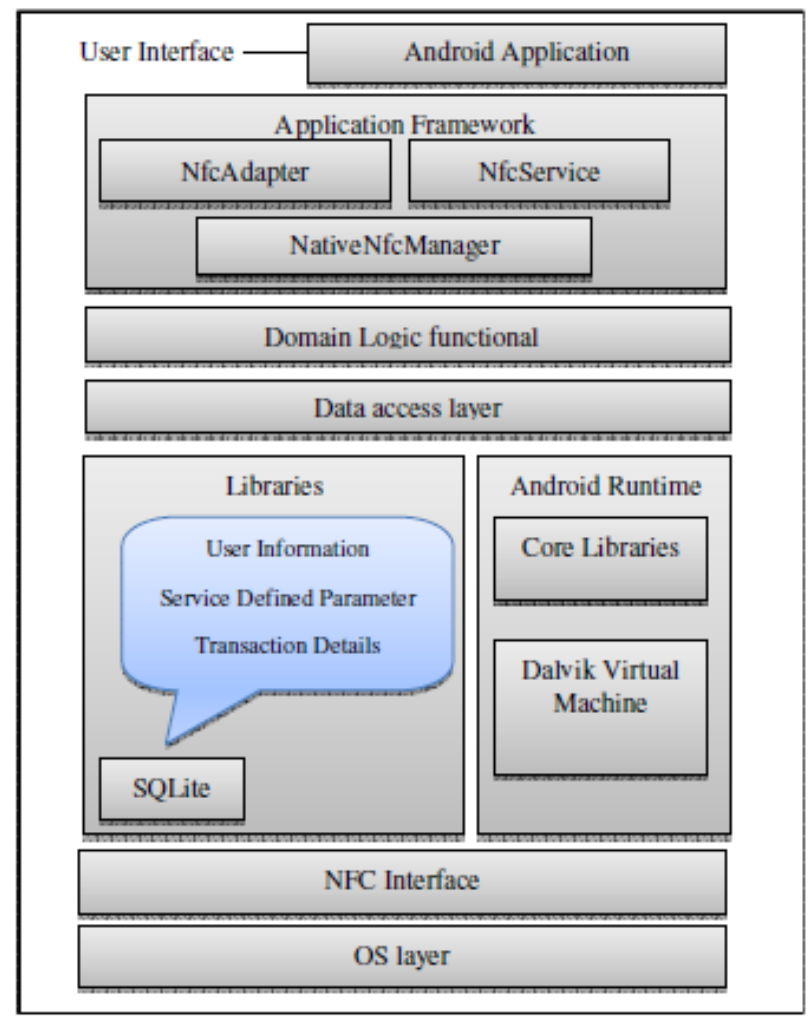

Figure1. NFC Smartphone Application Software Architecture

\section{b) Locker Generate System:}

Locker Generate System is located at each store which comprises of NFC reader connected to the seller's system via USB interface. When asked to generate a locker, seller needs to enter number of packets (purchased by customer) and send this information to the shopping mall sever (database) which will, in response, generate locker number(s) depending upon the number the packets. Lockers can be of different types based on the requirement by a particular store. Shopping mall database has the complete information of the lockers available at the locker system. Cash memo id is assigned to the assigned lockers in the database of shopping mall and can be referred in case of any error while collecting the packets. After generating the locker number(s), user is asked to tap smartphone to the NFC reader placed on the counter. NFC reader writes the data (locker number) to the NFC tag built in the smartphone. If the data has been written successfully, popup message will come on screen showing the detailed information. Database will maintain a status of each entry with status as "In Store" which mean the packets are there in the central locker system of shopping mall. 


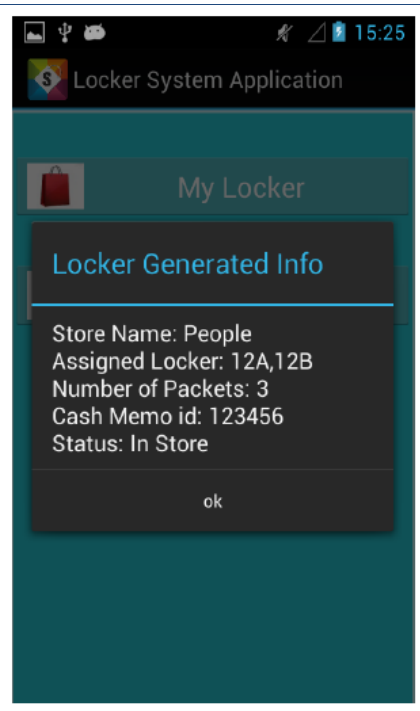

Figure 2. Information popup and stored in smartphone application database at the time of locker generation at store checkout counter

\section{c) Parcel Dispatch Station:}

Parcel dispatch station is located in the central locker system at the entrance or exit of mall which is used when the customer would like to get back his/her purchased items stored in the locker system. The dispatch station will have NFC reader connected to the system via USB interface. The NFC reader is used to read the information from the NFC tag built in the smartphone. When customer taps on it, the NFC reader only read the data whose status is "In Store" which is a valid data for shopping mall database and the data will be send to the shopping mall database which will in turn displays the complete information on screen. Status now of each entry change to "Delivered" once it is verified successfully by customer. While tapping, customer gets all the details on the UI showing "Verify and Exit Session" button. On pressing the button, the database in mobile is also get updated.

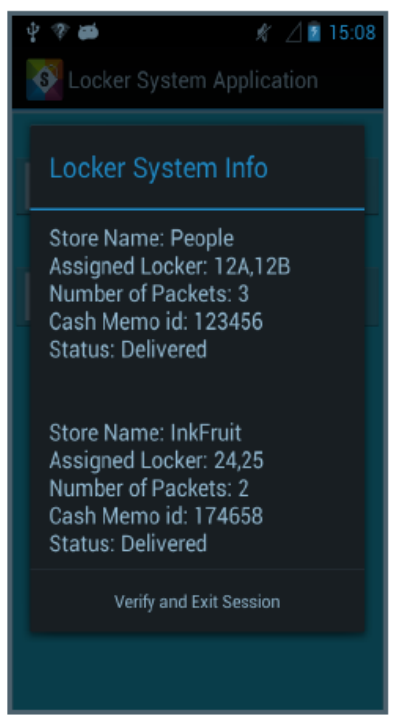

Figure 3. Information popup and stored in smartphone application database at the time of collecting packet from Parcel Dispatch Station 


\section{Service Outline}

The service outline for this proposed solution is described in five steps as follows:

Step 1: At the time of billing an item from a store, seller will ask the customer to avail the facility of locker system. If customer agrees to use the facility, Seller will enter the information (like the number of packets of the purchased item) and send it to the shopping mall server (database) which has the information about the available lockers at the parcel dispatch station and will generate a locker number depending on the packet classification and the number of packets. Corresponding to the generated locker number, details of number of packets, cash memo id and status will be stored in the shopping mall database. The status information would be set as "In Store", which means the packets are stored in parcel dispatch station.

Step 2: Customer will be asked to tap the NFC enabled smartphone to the reader placed at the checkout counter of the store. It will automatically activate the locker system application, which is specifically developed for this service, if it is pre-installed on the smartphone or else it will show the direction to the customer to download and install the application using Wi-Fi.

Step 3: While tapping on the reader, the reader writes the details of locker number on the application in the phone as well as displays it on the screen.

Step 4: After locker number gets stored in the phone and the details are verified by the customer, seller will deliver the packets to the parcel dispatch station and customer can move ahead to other stores for further shopping.

Step 5: When the customer approaches the parcel dispatch station while exiting the mall, customer will be directed to touch the smartphone on the reader placed at the counter; reader will then read the captured data from the application and send it to the database for verification. Database in return sends the information about the valid entries and displays it on the screen. The displayed data will have cash memo id and all the locker numbers assigned to the customer from all the stores of the shopping mall for the purchased items. Now this time the status of the items is updated from "In Store" to "Delivered". Customer can collect the packets and verify the items. In case of any mismatch, cash memo id can be used as a reference to track the item.

Special Case: There can be a situation when customer mobile device may be temporarily unoperational. For e.g., the battery may be drained or the mobile application is not responding or even the NFC reader at the parcel dispatch station has some issue. In such cases customer can use the cash receipts of purchased items to retrieve them back from parcel dispatch station. After collecting the packets, the "Status" information of the entries in the shopping mall database is updated from "In Store" to "Delivered". But the status of the corresponding entries in the customer's mobile application database will be unchanged as "In Store". So, the next time customer uses his mobile phone to avail locker facility and use the mobile device to retrieve packets from parcel dispatch station, the database of mobile device application is updated and bring in sync with the shopping mall database. Alternatively, Customer can even update his mobile database later on while connecting it to the server. 


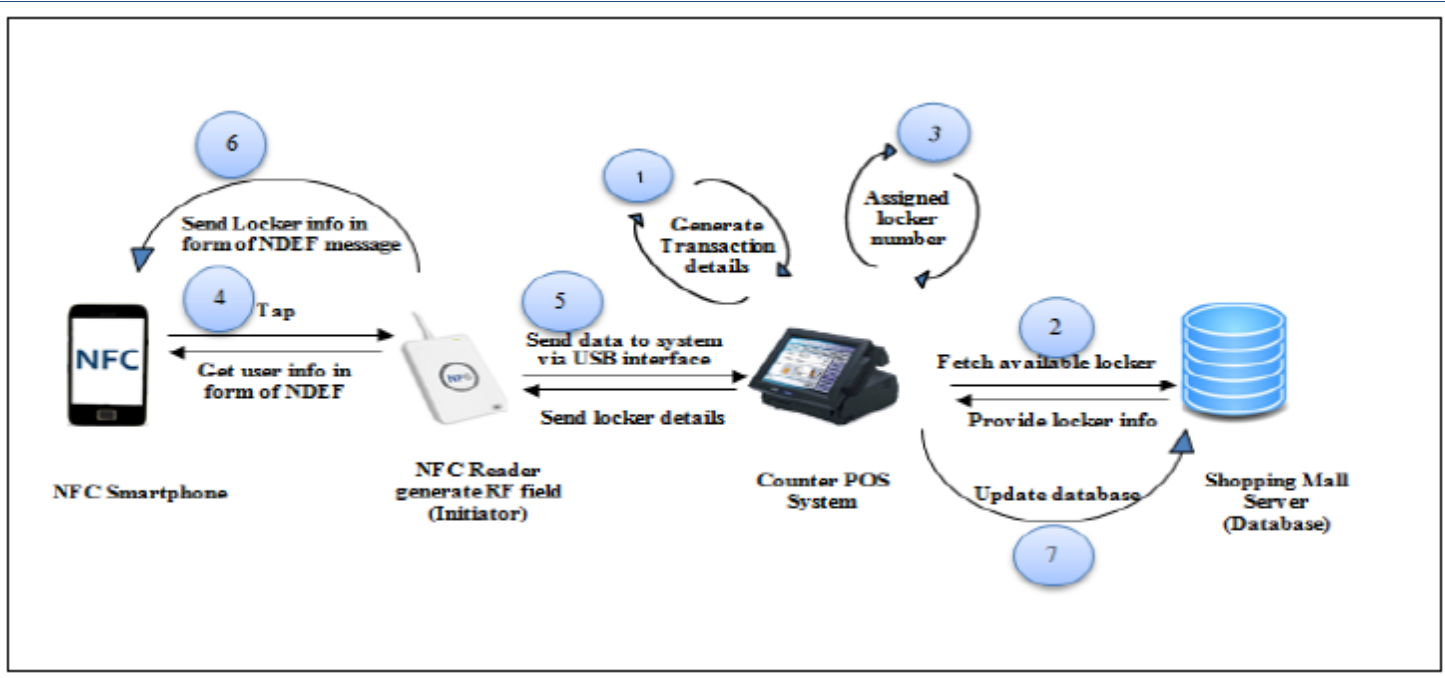

Figure 4. Solution Architecture- Locker Generation at store checkout counter

There are several benefits to customers of using this central locker system solution in shopping mall:

- Security of their purchased products.

- Organization (no more token lost!).

- Record Keeping.

- Minimize time consumed for depositing packets at store locker system while entering or exiting store.

- Eliminate chances of even losing purchased item inside mall.

- Get rid of the overhead of carrying packet in hand while roaming inside the mall.

As for the shopping mall retailers, it offers few useful benefits:

- NFC reader can be easily integrated with any POS (point of sale) system.

- Reduce Locker system installation at each store.

- Reduce Man-power also.

- Provide better service quality to customer.

- Allow them to convert their casual customers to loyal customers.

\section{Conclusion}

Customers like to visit stores that offer something different. If it is something that makes life more convenient for them, then they are more likely to revisit such a store [12]. This is exactly NFC technology offers in this paper. This paper presents an easy and convenient solution to customers for not carrying any purchased items in hand and storing it safely, while inside the mall. It has a very minimal customer interaction only, i.e., just a tap on the NFC reader. It will help to keep the track of items purchased from the shopping mall. It involves a lot of work for the shopping mall management at the backend to maintain the database and to have a large locker system area with the locker system of each and every store maintained separately at central locker system to easily retrieve the stored item back. It also poses a challenge to deliver packet from store to parcel dispatch station. The design of central locker system 
as well as delivering of packet to dispatch station is completely shopping mall specific. For customer, it's just to download the application in their smartphone and use it while stepping inside the mall. This would even show many advantages to business sector also in terms of cost reduction, reducing man power, better space utilization, i.e., one central locker system instead of having locker system as each and every counter. This solution presents a win-win situation for both retailers and customers as far as benefits are concerned. For future work, this solution can be further used to support marketing and promotions. It can be used to integrate coupons, offers and loyalty programs which can be sent to customer's smartphone while using this service based on their past purchases. It does not require any other customer signup or registration or any overhead. With the emergence of NFC technology in Smartphones and its applications in the service sector business; this solution can further improve the usability of these systems and provide flexibility to the shopping mall management system to improve service experiences using the solution framework.

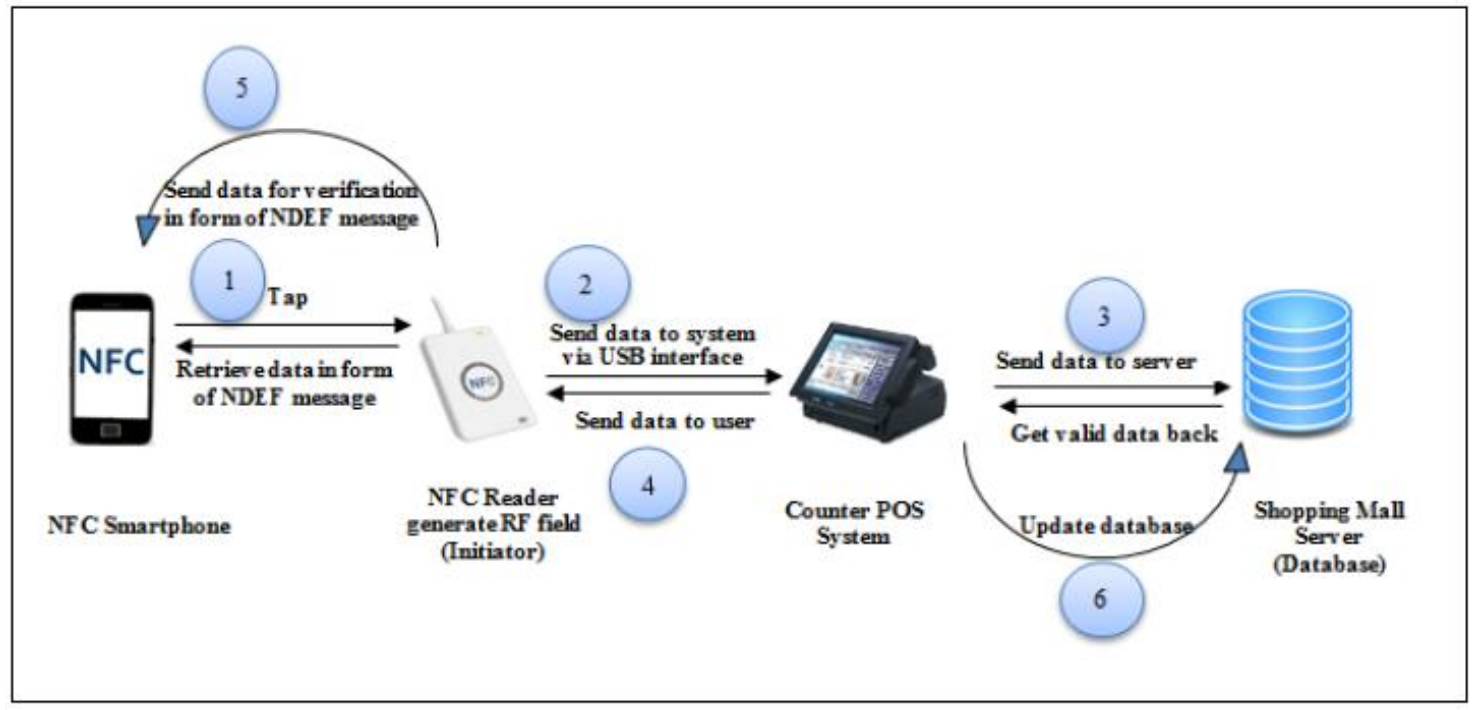

Figure 5. Solution Architecture- Parcel Dispatch Station

\section{ACKNOWLEDGMENT}

I would like to extend my sincere thanks to Mr. Pritam Bhowal, Lead Engineer, Samsung Research Institute-Bangalore (SRIB) for his kind co-operation in analyzing the key features regarding the project and provide necessary information. Many thanks and appreciations to him for helping me in completion of this paper.

\section{REFERENCES}

[1]. Shopping, http://en.wikipedia.org/wiki/Shopping [referenced 20th May, 2014]

[2]. Near Field Communication, http://en.wikipedia.org/wiki/Near_field_communication [referenced 25th May, 2014] 
Siddarth Poddar; Central Locker System for shopping mall using NFC Based Smartphone, Transactions on Networks and Communications, Volume 2 No 5, Oct (2014); pp: 156-164

[3]. Indoor Navigation System, http://ieeexplore.ieee.org/xpl/login.jsp?tp=\&arnumber=5954491\&url=http\%3A\%2F\%2Fieeexplore.ieee.org \%2Fstamp\%2Fstamp.jsp\%3Ftp\%3D\%26arnumber\%3D5954491

[4]. Mobile Payment, Components for an interoperable NFC mobile payment ecosystem http://ieeexplore.ieee.org/xpl/mostRecentlssue.jsp?punumber=6478871 [referenced 17th July,2014]

[5]. NFC Reader, http://www.acs.com.hk/en/products/3/acr122uusb-nfc-reader/ [referenced 18thMay 2014]

[6]. http://businesstoday.intoday.in/story/near-fieldcommunications-nfc/1/21671.html [referenced 18th June 2014]

[7]. http://randomoracle.wordpress.com/2013/12/02/nfc-cardemulation-and-android-4-4-part-i/ [referenced 17th July, 2014]

[8]. NDEF technical Specification, http://www.eetchina.com/ARTICLES/2006AUG/PDF/NFCForum-TSNDEF. pdf?SOURCES=DOWNLOAD [referenced 18th June 2014]

[9]. https://developer.android.com/guide/topics/connectivity/nfc/hce.html [referenced 17th July, 2014]

[10]. http://rapidnfc.com/nfc_enabled_phones [referenced 18th June 2014]

[11]. Android Developers, http://developer.android.com/index.html.

[12]. http://www.geekbusiness.com/2012/10/let-your-retail-businesstake-advantage-of-nfc-technology 